\title{
Recent advances in the research for the homolog of breast cancer associated gene AtROW1 in higher plants
}

\author{
Yue Jiao $^{1,2}$, Yuzhou Zhang ${ }^{1} \&$ Yu-Xian Zhu ${ }^{1 *}$ \\ ${ }^{1}$ State Key Laboratory of Protein and Plant Gene Research, College of Life Sciences, Peking University, Beijing 100871, China; \\ ${ }^{2}$ Center for Science and Technology Development, Ministry of Agriculture, Beijing 100176, China
}

Received April 27, 2016; accepted May 30, 2016

\begin{abstract}
BARD1 (BRCA1 associated RING domain protein 1), as an important animal tumor suppressor gene associated with many kinds of cancers, has been intensively studied for decades. Surprisingly, homolog of BARD1 was found in plants and it was renamed AtROW1 (repressor of Wuschel-1) according to its extremely important function with regard to plant stem cell homeostasis. Although great advances have been made in human BARD1, the function of this animal tumor-suppressor like gene in plant is not well studied and need to be further elucidated. Here, we review and summarize past and present work regarding this protein. Apart from its previously proposed role in DNA repair, recently it is found essential for shoot and root stem cell development and differentiation in plants. The study of AtROW1 in plant may provide an ideal model for further elucidating the functional mechanism of BARD1 in mammals.
\end{abstract}

repressor of Wuschel-1, DNA repair, stem cell niche

Citation: Jiao, Y., Zhang, Y., and Zhu Y.X. (2016). Recent advances in the research for the homolog of breast cancer associated gene AtROW1 in higher plants. Sci China Life Sci 59, 825-831. doi: 10.1007/s11427-016-5086-6

\section{INTRODUCTION}

Breast and ovarian cancer are major causes of mortality and morbidity in the developed world. A high percentage of breast cancers are due to the inheritance of germ line mutations in two breast cancer susceptibility loci, BARD1and its homologue BRCA1 (Elledge and Amon, 2002; Westermark et al., 2003). As a tumour suppressor gene, two regions of the BRCA1/BARD1 protein are thought to be important for its function: an N-terminal RING domain involved in DNA repair (Fabbro et al., 2002; Williams et al., 2004) and the C-terminal BRCT domain that mediates a specific interaction with phosphorylated forms of DNA repair factors (Baer and Ludwig, 2002). BRCA1 is able to interact with BARD1 (BRCA1 associated RING domain protein 1) to form a heterodimer through their common $\mathrm{N}$-termini, which plays

*Corresponding author (email: zhuyx2@pku.edu.cn; zhuyx@whu.edu.cn) important roles in many life processes, including cell cycle control, DNA repair, DNA recombination and transcriptional regulation (Wu-Baer et al., 2003; Feng et al., 2009).

It was quite a surprise to find homologs of the human BRCA1 and BARD1 in seed plants. The genes located on Arabidopsis chromosome 1 (At1g04020) and 4 (At4g21070) with almost identical BRCT and RING domain structures were separately identified and named $A t$ $B R C A 1$ and AtBARDI, respectively, with similar functions in DNA repair initially (Lafarge and Montane, 2003; Reidt et al., 2006). However, further work has revealed that AtBARD1 was involved in maintaining shoot apical meristem development by suppressing WUS (Wuschel) expression out of the organizing centre (OC) (Han et al., 2008). Therefore $B A R D 1$ in plant was renamed as ROWI (repressor of WUS-1) (Han and Zhu, 2009). Recent work showed that ROW1 is also essential for the maintenance of quiescent centre (QC) and root stem cell niche development through 
repressing WOX5 expression in root proximal meristem above the QC (Zhang et al., 2015). Down-regulation of WOX 5 expression partially rescues the stem cell niche defect in row1-3 mutant (Drisch and Stahl, 2015; Kong et al., 2015). Here we summarize AtROW1 functions in plant shoot and root stem cell development.

\section{ATROW1 AND ITS HOMOLOGS IN PLANTS}

BARD1 and BRCA1 are homologous proteins, which suppress tumour genesis in animals and is involved in many cellular processes including DNA repair as heterodimers formed upon ubiquitination. The Arabidopsis homolog genes, At4g21070 and At1g04020, contain the N-terminal RING domain and the C-terminal BRCT domains with no substantial similarities of other motifs compared with their counter parts in animals (Figure 1). In contrast to mammalian mutations, two of the original Arabidopsis T-DNA insertion mutant lines showed no apparent disorders in embryogenesis with almost normal plant development (Lafarge and Montane, 2003; Reidt et al., 2006). These mutant lines thus provide us ideal models for studying their biological or biochemical functions in the plant kingdom.

\section{Identification of AtROW1 in plants}

AtBRCA1, first reported in 2003, encodes 941 amino acids with molecular weight of about $104 \mathrm{kD}$. Compared with human BRCA1, AtBRCA1 contains one extra P300/CBP domain besides the two commonly conserved RING domain and BRCT domains. Bioinformatics analysis reveals that AtBRCA1 does not contain the coiled-coil domain as found in the human gene. AtROW1, first reported in 2006 as AtBARD1 (Reidt et al., 2006), encodes 713 amino acids with no Ankyrin (ANK) domain (Figure 1). Yeast two-hybrid experiment showed that ROW1 was able to directly interact with AtBRCA1 in vivo. Further experiments revealed that the N-terminal fragment of AtBRCA1 interacted with full length ROW1, while the C-terminal part of AtBRCA1 could not bind to full length AtROW1. Also, AtBRCA1 does not interact with the C-terminal fragment of AtROW1. Therefore it is concluded that AtBRCA1 interacts with AtROW1 through their N-terminal domains (Reidt et al., 2006; Han et al., 2008).

The RING domain of human BRCA1 contains two nuclear export signal peptides and one nuclear localization signal peptide. We do not know whether these signal peptides are present in the Arabidopsis gene yet. In human, BARD1 distributes widely in both the cytoplasm and in nucleus (Chen et al., 1996). The nuclear translocation of hBRCA1 is achieved via two pathways: a classical pathway that recruits importin $\alpha / \beta$ proteins whereas the other pathway involves binding of hBARD1 to initiate transport processes (Chen et al., 1996). When hBRCA1 binds hBARD1 via their RING domains, the nuclear export signal located in the N-termini are blocked to prevent their escaping from the nucleus (Fabbro et al., 2002). Split-YFP experiment in plants revealed that AtBRCA1 and ROW1 heterodimer is also localized in the nucleus (Reidt et al., 2006).

SMART, a domain-finding software, analysis showed that AtBRCA1 contained a PHD domain (plant homeodomain) encoding about 66 amino acids located between the P300/CBP domain and the BRCT domain, which is absent in animals. PHD-containing proteins were known to localize in the nucleus (Bienz, 2006). The conserved motif of the PHD domain is $\mathrm{C} 4 \mathrm{HC} 3$, which specifically bind trimethylated histone $\mathrm{H} 3$ lysine $4(\mathrm{H} 3 \mathrm{~K} 4 \mathrm{me} 3)$ to regulate target gene transcription (Li et al., 2006; Shi et al., 2006; Sung and Amasino, 2004; Wysocka et al., 2006; Cui et al., 2013; Lu et al., 2011). ROW1 also contains the PHD domain (Han and Zhu, 2009). Amino acid sequence analysis indicates that, the PHD domains of AtBRCA1 or ROW1 are conserved to higher degrees than that of the RING and the BRCT domains (Trapp et al., 2011, Han and Zhu, 2009).

\section{The evolution and origin of BARD1 and its homolog in plants}

A phylogenetic tree showed that the animal and plant BRAD1/BRCA1 share a common eukaryotic ancestor before their divergence (Figure 2). It was widely speculated that, after the separation of plant and animal kingdoms, a common ancestor that gave rise to both BRCA1 and BARD1 proteins in plant subsequently gained a PHD domain (Trapp et al., 2011; Yoon et al., 2004; Hedges et al., 2006). After that, a duplication event separately took place in plant and animal systems, which produces these two homologous proteins that lack the PHD domain in mammals. The appearance of the PHD domain can be dated back to about 1,150 million years ago, during the origin of green algae (Yoon et al., 2004; Hedges et al., 2006). Further analysis showed that the duplication event that produced

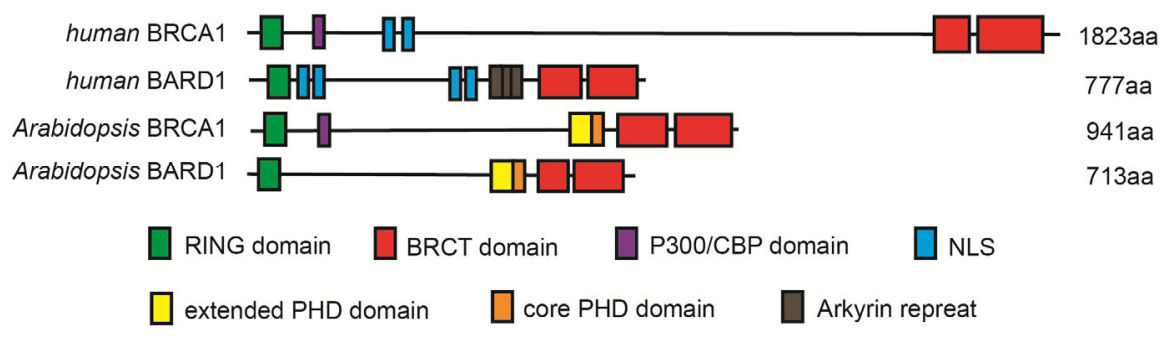

Figure 1 Comparisons of the human BARD1 and BRCA1 with their homologue from Arabidopsis. 


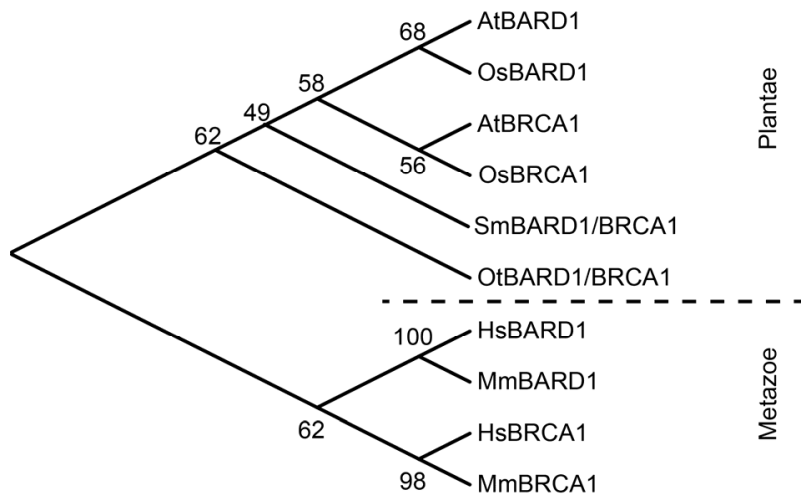

Figure 2 The evolution and diversification of BARD1 and its homologous BRCA1 in plants and animals. The BRRD1/BRCA1 phylogenetic tree was constructed by using the Minimum Evolution methods. At, Arabidopsis thaliana; Os, Oryza sativa; Sm, Selaginella moellendorffii; Ot, Ostreococcus tauri; $\mathrm{Mm}$, Mus musculus; $\mathrm{Hs}$, Homo sapiens.

BRCA1 and BARD1 proteins in the plant kingdom may took place after the divergence of Lycopodiopsida and Spermatophytina (seed plant), earlier than the time that monocotyledons and dicotyledons separated, because homologs of BRCA1 and BARD1 were found in both the two late categories (Soltis et al., 2002; Zimmer et al., 2007). Alternatively, the ancestor of these two proteins in plant and animal might possess the PHD domain to begin with. It is after the separation of these two major lineages, the PHD domain in the animal protein was lost while it is retained in plants. However this hypothesis is challenged, for both BRCA1 and BARD1 proteins in green algae Volvox and Chlamydomonas do not contain the PHD domain (Faucher and Wellinger, 2010).

\section{ATROW1 IS INVOLVED IN DNA REPAIR IN ARABIDOPSIS}

DNA damage imposes continuous threat to genomic integrity in eukaryotic cells (Hartung et al., 2007). DNA double-strand break (DSB) is the most serious form of DNA damage that can be lethal to a cell. Cells normally repair DSBs by two approaches, homologous recombination (HR) and nonhomologous end joining (NHEJ) (Boulton, 2006). HR precisely restores the integrity of a broken DNA using an intact and homologous DNA strand as the template, while NHEJ is an unprecise repair process in which the ends of a DSB might be modified.

\section{BARD1 is important for DNA repair in animals}

BARD1 is one of the important factors to promote genome stability in animals, and it coordinates DNA-damage responses. Studies on a BARD1 homologue in Caenorhabditis elegans showed that loss of BARD1 protein resulted in germination defects and radiation sensitivity (Westermark et al., 2003). Mutated HsBRCAl or HsBARDl genes led to hypersensitivity against DNA damaging agents such as mitomycin C (MMC), which cross links DNA (Swoboda et al., 1994).

\section{AtROW1 participates in DNA repair in Arabidopsis}

Three Arabidopsis rowl T-DNA mutant lines have been identified (Figure 3). row1-1 (SALK_097601) has a T-DNA inserted in the first intron of the gene while the second insertion (SALK_031862, row1-2) is located in the third exon, which codes for the N-terminal RING domain. Real-time quantitative PCR (RT-qPCR) results indicated that rowl-1 and row 1-2 mRNA levels were reduced to 39\% and $46 \%$, respectively, of the wild-type level. A third T-DNA (row1-3, SALK_003498), is inserted in the last exon of the gene that resulted in a complete blockage of AtROWI expression. In comparison to wild-type plants, row $1-1$ and rowl-2 homozygous line do not show visible phenotype when grown under normal conditions. However, when challenged with the DNA cross-linking agent MMC, these two insertion mutants displayed a more sensitive phenotype as compared to wild-type seedlings (Han et al., 2008). Using a substrate that promotes DNA recombination, both Atrowl and Atbrcal mutants were shown to have defects in $\mathrm{HR}$, which was especially prominent after the introduction of DSBs (Wu-Baer et al., 2003; Reidt et al., 2006; Block-Schmidt et al., 2010). To elucidate whether both these proteins act in the same DNA repair pathway, brcal-1 mutant was crossed with row 1-2 to create a row 1-2/brcal-1 double mutant to quantify the degree of MMC sensitivity, in comparison with both single mutants. The double mutant was no more sensitive than each of the single mutant, indicating that AtBRCA1 and AtROW1 are epistatic for DNA cross-link repair (Reidt et al., 2006). UV-C recovery assay and terminal transferase dUTP nick end labeling (TUNEL)-based in situ cell death analysis showed obvious defects in DNA repair in rowl-3 seedlings, indicating that ROW1 plays a very important role in DNA repair in plants (Han et al., 2008).

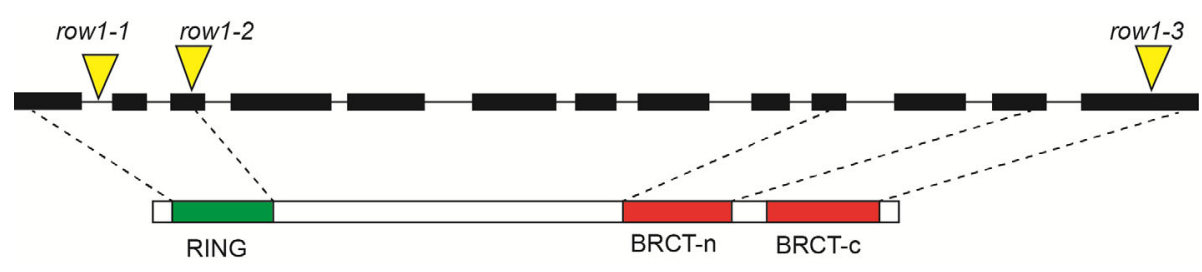

Figure 3 Analysis of the T-DNA insertion sites for three AtROW1 mutant lines identified from the Salk institute. 


\section{AtROW1 may form a complex with AtBRCA1 and other factors for DNA repair}

BARD1 forms a heterodimer with BRCA1 in human. The BARD1-BRCA1 heterodimer recruits other different repair factors to form three complexes, named A, B and C, respectively, required for proper DNA repair. The homologs of all these animal DNA-damage repair factors that interact with the BRCT domain at the $\mathrm{C}$ terminus of BRCA1, are found in Arabidopsis (Table 1). AtBRCC36A and AtBRCC36B were found to share greater than $90 \%$ sequence identity with that of the human BRCC36 (Cooper et al., 2009; Block-Schmidt et al., 2010; Cantor and Xie, 2010). Homologs of MLH1 and MSH6, which is essential for formation of complex B in animals, was also identified in Arabidopsis (Ade et al., 1999; Jean et al., 1999; Greenberg et al., 2006). Likewise, MRE11, RAD50 and NBS1, essential components for complex C, were highly conserved in both human and Arabidopsis (Daoudal-Cotterell et al., 2002; Bleuyard et al., 2004; Gallego et al., 2001; Waterworth et al., 2007). These results suggest that AtROW1 may play a role in plant DNA repair by using a similar mechanism compared with animals.

\section{ATROW1 MAINTAINS APICAL STEM CELL DEVELOPMENT IN PLANTS}

Plant stem cells, contained in specialized structures called meristems, sustain a constant supply of cells that enable plants to grow and produce new organs throughout their life spans (Scheres, 2007), which are mainly confined within the shoot apical meristem (SAM) and root apical meristem (RAM) (Heidstra and Sabatini, 2014). OC and QC localized in SAM and RAM, respectively, play important roles in maintaining their stem cell populations by creating special microenvironment (Weigel and Jürgens, 2002). WUS- 1 and WOX5, the most important conserved factor that regulate shoot and root stem cell organization, are specifically expressed in OC and QC, respectively (Dinneny and Benfey, 2008; Busch et al., 2010). WUS establishes the shoot stem cell niche in the SAM through a CLAVATA3 (CLV3)WUS feedback loop, whereas WOX5 establishes the root stem cell niche in the RAM by a feedback circuit involving auxin-related response factors (Su et al., 2009, 2011; Xu et al., 2013; Song et al., 2012). AtROW1, a PHD-domain containing protein, is a key repressor to maintain both the SAM and RAM structures by interacting with WUS- 1 and WOX5 independently (Han et al., 2008; Zhang et al., 2015).

\section{AtROW1 maintains the shoot apical meristem by con- fining WUS-1 expression to OC}

In plants, all above-ground tissues develop from stem cells located in SAM (Gaillochet et al., 2015). Severe SAM defects were observed in row 1-3 mutant shoot, and WUS-1 transcripts accumulated over 200-fold as compared to the wild type by RT-qRCR and other analyses. In situ hybridization experiments showed that WUS- 1 was released to the outmost cell layers above the OC (Figure 4). Besides, wus-1/row1-3 double mutant showed prematurely terminated SAM structures identical to those of wus-1. Similar to wus-1 phenotype was observed in AtROWl overexpression lines where WUS-1 transcript levels were significantly reduced. These results suggest that the defective SAM of row 1-3 mutant may be caused by WUS-1 overexpression. Either full-length AtROWI or a construct encoding its C-terminal domain (ROW1:C-ter;row1-3) was sufficient to complement the rowl-3 phenotype, indicating that AtROW1 function relies on its C-terminus. Gel shift assay showed that a specific WUS-1 promoter region was recognized by nuclear protein extracts obtained from wild-type plants, and this protein-DNA complex was recognized by antibodies against ROW1 (Han et al., 2008).

Co-immunoprecipitation (CoIP) assays indicated that AtROW1 may function together with SYD, a SWI-SNF2 ATPase subunit of the chromatin remodeling complex (Yang et al., 2015; Fu et al., 2016), which is specifically recruited to the WUS-1 promoter (Han et al., 2008). It was thus proposed that AtROW1 inhibits chromatin remodeling through the PHD domain and that this process is necessary for WUS-1 expression. We therefore conclude that AtROWI regulates SAM organization and maintenance by limiting WUS-1 expression to the OC.

Table 1 Comparison of the homologous proteins that interact with the BRCA1-BARD1 heterodimer to form the BRISC complex that participates potentially in DNA-damage repair

\begin{tabular}{lcccr}
\hline Protein & Human accession No. & Length (aa) & Arabidopsis accession No. & Length (aa) \\
\hline BRCA1 & NP_009225.1 & 1,823 & AT4G21070 & 941 \\
BARD1 & NP_000456.2 & 777 & AT1G04020 & 713 \\
BRCC36 & NP_077308.1 & 316 & AT1G80210(BRCC36A) & 406 \\
& & & AT3G06820(BRCC36B) & 405 \\
MLH1 & NP_000240.1 & 520 & AT4G09140 & 737 \\
MSH6 & NP_000170.1 & 961 & AT4G02070 & 1,324 \\
MRE11 & NP_005582.1 & 708 & AT5G54260 & 720 \\
RAD50 & NP_005723.2 & 1,312 & AT2G31970 & 1,316 \\
NBS1 & NP_002476.2 & 754 & AT3G02680 & 542 \\
\hline
\end{tabular}




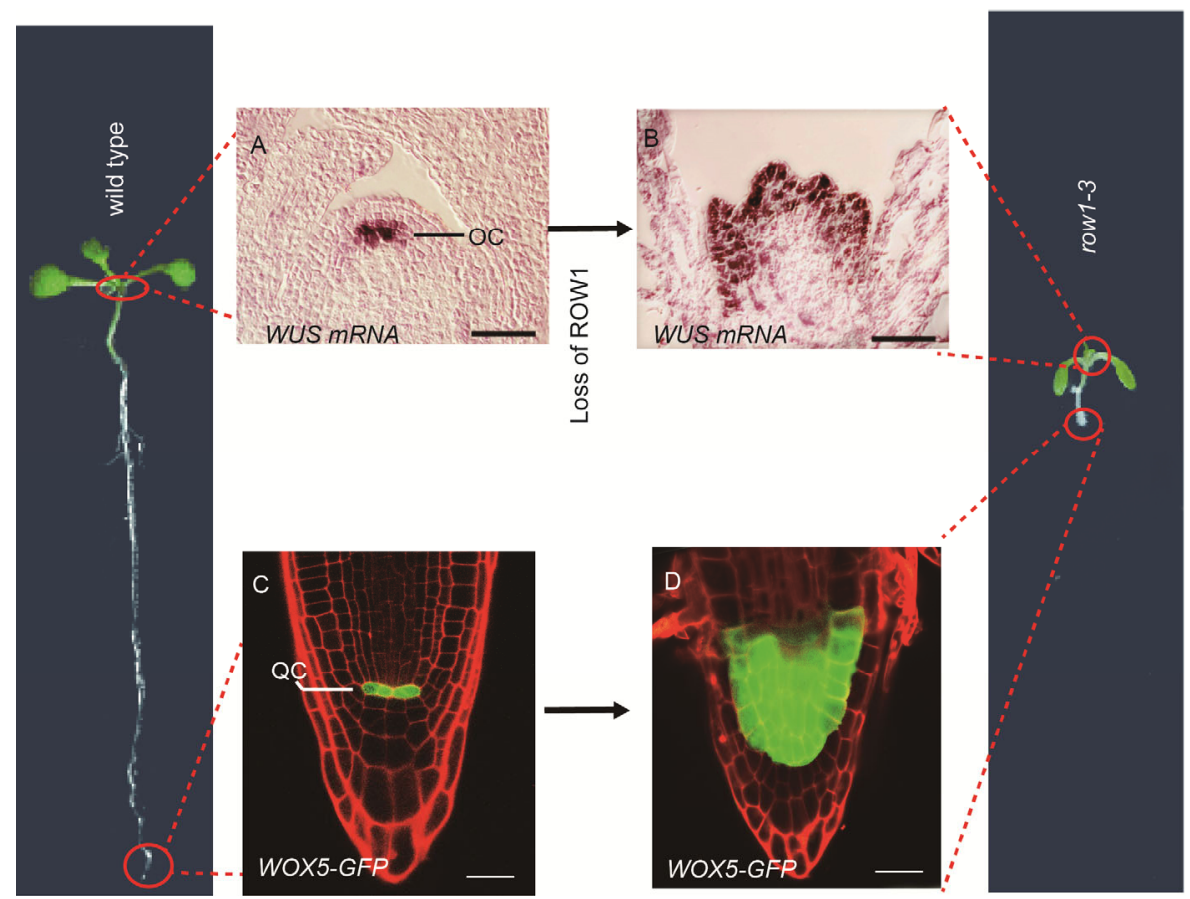

Figure 4 AtROW1 maintains Arabidopsis shoot and root apical meristem development by confining the WUS and WOX5 expression within the OC and QC, respectively (modified from Han et al., 2008; Zhang et al., 2015). Scale bars, $20 \mu \mathrm{m}$.

\section{AtROW1 maintains the root apical meristem by confin- ing WOX5 expression to the QC}

QC in Arabidopsis RAM orchestrates a fine balance of the stem cell pool (Heyman et al., 2013; Zhou et al., 2015; Xu, 2015). In row1-3 knockout mutant, we observed severe root architecture defects, including extremely short roots and complete loss of gravitropism. RT-qPCR and GFP-fusion assay indicated that loss of AtROW1 function led to drastic WOX5 over-expression in cells that normally express AtROW1 (Figure 4). As a result, the mutant has no obvious QC structure with no proximal or distal meristem cell differentiation. A root phenotype similar to that of the loss-offunction mutant wox5-1 was observed in wox5-1/row $1-3$ double mutant, indicating that AtROW1 is genetically epistatic to WOX5. Further experiments showed that AtROW1 bound specifically to trimethylated histone H3 lysine 4 (H3K4me3) located in the WOX5 promoter region, consistent with a function in transcriptional repression. When ROW1 was aberrantly expressed in QC, WOX5 expression was reduced to undetectable levels with a resultant root phenotype identical to that of wox5-1, including premature differentiation of the distal stem cell (DSC) layer and disordered columella cell arrangement. This confirms the notion that AtROW1 is essential for normal stem cell niche development and for maintenance of QC identity in Arabidopsis through direct repression of WOX5 in the proximal meristem. In the wild-type background, WOX5::GFP signals disappeared after 3 days of auxin treatment, whereas no such repression was observed in wild-type Arabidopsis expressing the ROW1::GFP construct or in row $1-3$ mutant expressing the WOX5::GFP construct, after the same auxin treatment. Thus we suggest that AtROW1 may regulate WOX5 expression downstream of auxin signaling (Zhang et al., 2015; He and Zhao, 2015). All these results seem to support a conclusion that AtROW1 is a key repressor required for maintenance of both SAM and RAM identity by interacting with WUS-1 and WOX5, two master regulators of Arabidopsis stem cell development.

\section{PERSPECTIVES}

Tumor suppressors are known to regulate the size of the stem cell niche in animals by controlling cell cycle and cell differentiation (Sage, 2012). A tumor suppressor protein ING2, which is a PHD domain-containing protein, represses target gene transcription by binding to $\mathrm{H} 3 \mathrm{~K} 4 \mathrm{me} 3$ histone markers to suppress tumor formation (Shi et al., 2006). AtROW1, another tumor suppressor-like gene, was found to maintain the stem cell niche in plants. Complete loss of AtROW1 function resulted in huge root cell masses similar to tumor formation in animals (Han et al., 2008). A homozygous mutation in bardl exhibits embryonic lethality in the animal model that prevented full elucidation of this gene function over the whole life cycle. We may overcome this problem in Arabidopsis because homozygous Atrowl mutant is viable and will likely providing a valuable alternative system for in-depth mechanistic studies of this important gene family. Also, quite a few DNA-damage repair factors have been identified in animals and in plants through simple sequence alignment, we suggest to use AtROW1-interacting 
factors to search for DNA repair machineries in higher plants.

Quite a number of outstanding issues related to either AtROW1 function or to its regulatory mechanisms have yet to be addressed. For example, we know nothing about how and by whom AtROWI was controlled in terms of transcription or translation. It is also not clear as to whether AtROWI manipulate WUS-1/WOX5 expression in OC and QC, respectively, via a similar or different mechanism. Furthermore, we know that WUS-1 is not expressed above the OC in Arabidopsis SAM and WOX5 is not expressed above the QC in the RAM because the existence of AtROW1 prevented target gene expression in these areas. Why both genes failed to be transcribed in regions beneath the $\mathrm{OC}$ and QC, where AtROW1 is not expressed? Apart from WUS-1 and WOX5, are there any other potential target genes of AtROW1? Are there new and important AtROW1-interacting factors for regulations of stem cell niche or for DNA repair?

TEs may establish and rewire gene regulatory networks by genetic and epigenetic mechanisms to modulate stem cell development (Wei and Cao, 2016; Wang et al., 2016). In human embryonic stem cells, about $20 \%$ of the transcription factor binding sites were reported to be associated with TE insertions (Kunarso et al., 2010). Transcription factor binding activities were altered significantly in row 1-3 mutant with respect to WUS-1 expression (Han et al., 2008). A systematic search for TEs in genes related to Arabidopsis stem cell niche development may help us to solve the puzzle via a different angle. We anticipate that functional studies with regard to AtROWI and related genes may become a hot topic in the near future.

Compliance and ethics The author(s) declare that they have no conflict of interest.

Acknowledgements This work was supported by the National Natural Science Foundation of China (90717009).

Ade, J., Belzile, F., Philippe, H., and Doutriaux, M.P. (1999). Four mismatch repair paralogues coexist in Arabidopsis thaliana: AtMSH2, AtMSH3, AtMSH6-1 and AtMSH6-2. Mol Gen Genet 262, 239-249.

Baer, R., and Ludwig, T. (2002). The BRCA1/BARD1 heterodimer, a tumor suppressor complex with ubiquitin E3 ligase activity. Curr Opin Genet Dev 12, 86-91.

Bienz, M. (2006). The PHD finger, a nuclear protein-interaction domain. Trends Biochem Sci 31, 35-40.

Bleuyard, J.Y., Gallego, M.E., and White, C.I. (2004). Meiotic defects in the Arabidopsis rad50 mutant point to conservation of the MRX complex function in early stages of meiotic recombination. Chromosoma 113, 197-203.

Block-Schmidt, A.S., Dukowic-Schulze, S., Wanieck, K., Reidt, W., and Puchta, H. (2010). BRCC36A is epistatic to BRCA1 in DNA crosslink repair and homologous recombination in Arabidopsis thaliana. Nucleic Acids Res 39, 146-154.

Boulton, S.J. (2006). Cellular functions of the BRCA tumour-suppressor proteins. Biochem Soc Trans 34, 633-645.

Busch, W., Miotk, A., Ariel, F.D., Zhao, Z., Forner, J., Daum, G., Suzaki,
T., Schuster, C., Schultheiss, S.J., Leibfried, A., Haubeiss, S., Ha, N., Chan, R.L., and Lohmann, J.U. (2010). Transcriptional control of a plant stem cell niche. Dev Cell 18, 841-853.

Cantor, S.B., and Xie, J. (2010). Assessing the link between BACH1/FANCJ and MLH1 in DNA crosslink repair. Environ Mol Mutagen 51, 500-507.

Chen, C.F., Li, S., Chen, Y., Chen, P.L., Sharp, Z.D., and Lee, W.H. (1996). The nuclear localization sequences of the BRCA1 protein interact with the importin-alpha subunit of the nuclear transport signal receptor. J Biol Chem 271, 32863-32868.

Cooper, E.M., Cutcliffe, C., Kristiansen, T.Z., Pandey, A., Pickart, C.M., and Cohen, R.E. (2009). K63-specific deubiquitination by two JAMM/MPN+ complexes: BRISC-associated Brcc36 and proteasomal Poh1. EMBO J 28, 621-631.

Cui, X., Jin, P., Cui, X., Gu, L., Lu, Z., Xue, Y., Wei, L., Qi, J., Song, X., Luo, M., An, G., and Cao, X. (2013). Control of transposon activity by a histone H3K4 demethylase in rice. Proc Natl Acad Sci USA 110, 1953-1958.

Daoudal-Cotterell, S., Gallego, M.E., and White, C.I. (2002). The plant Rad50- Mre11 protein complex. FEBS Lett 516, 164-166.

Dinneny, J.R.,and Benfey, P.N. (2008). Plant stem cell niches: standing the test of time. Cell 132, 553-557.

Drisch, R.C., and Stahl, Y. (2015). Function and regulation of transcription factors involved in root apical meristem and stem cell maintenance. Front Plant Sci 6, 505.

Elledge, S., and Amon, A. (2002). The BRCA1 suppressor hypothesis: an explanation for the tissue-specific tumor development in BRCA1 patients. Cancer Cell 1, 129-132.

Fabbro, M., Rodriguez, J.A., Baer, R., and Henderson, B.R. (2002). BARD1 induces BRCA1 intranuclear foci formation by increasing RING-dependent BRCA1 nuclear import and inhibiting BRCA1 nuclear export. J Biol Chem 277, 21315-21324.

Faucher, D., and Wellinger, R.J. (2010). Methylated H3K4, a transcription-associated histone modification, is involved in the DNA damage response pathway. PLoS Genet 6, e1001082.

Feng, L., Huang, J., and Chen, J. (2009). MERIT40 facilitates BRCA1 localization and DNA damage repair. Genes Dev 23, 719-728.

Fu, X., Li, C., Liang, Q., Zhou, Y., He, H., and Fan, L.M. (2016). CHD3 chromatin-remodeling factor PICKLE regulates floral transition partially via modulating LEAFY expression at the chromatin level in Arabidopsis. Sci China Life Sci 59, 516-528.

Gaillochet, C., Daum, G., and Lohmann, J.U. (2015). O Cell, Where Art Thou? The mechanisms of shoot meristem patterning. Curr Opin Plant Biol 23, 91-97.

Gallego, M.E., Jeanneau, M., Granier, F., Bouchez, D., Bechtold, N., and White, C.I. (2001). Disruption of the Arabidopsis RAD50 gene leads to plant sterility and MMS sensitivity. Plant J 25, 31-41.

Greenberg, R.A., Sobhian, B., Pathania, S., Cantor, S.B., Nakatani, Y., and Livingston, D.M. (2006). Multifactorial contributions to an acute DNA damage response by BRCA1/BARD1-containing complexes. Genes Dev 20, 34-46.

Han, P., and Zhu, Y.X. (2009). BARD1 may be renamed ROW1 because it functions mainly as a REPRESSOR OF WUSCHEL1. Plant Signal. Behav 4, 52-54.

Han, P., Li, Q., and Zhu, Y.X. (2008). Mutation of Arabidopsis BARD1 causes meristem defects by failing to confine WUSCHEL expression to the organizing center. Plant Cell 20, 1482-1493.

Hartung, F., Suer, S., and Puchta, H. (2007). Two closely related RecQ helicases have antagonistic roles in homologous recombination and DNA repair in Arabidopsis thaliana. Proc Natl Acad Sci USA 104, 18836-18841.

Hedges, S.B., Dudley, J., and Kumar, S. (2006). Time Tree: a public knowledge-base of divergence times among organisms. Bioinformatics 22, 2971-2972.

Heidstra, R. and Sabatini, S. (2014). Plant and animal stem cells: similar yet different. Nat Rev Mol Cell Biol 15, 301-312.

He, Y., and Zhao, Y. (2015). A key link between jasmonic acid signaling and auxin biosynthesis. Sci China Life Sci 58, 311-312.

Heyman, J., Cools, T., Vandenbussche, F., Heyndrickx, K.S., Van Leene, 
J., Vercauteren, I., Vanderauwera, S., Vandepoele, K., De Jaeger, G., Van Der Straeten, D., and De Veylder, L. (2013). ERF115 controls root quiescent center cell division and stem cell replenishment. Science 342, 860-863.

Jean, M., Pelletier, J., Hilpert, M., Belzile, F., and Kunze, R. (1999). Isolation and characterization of AtMLH1, a MutL homologue from Arabidopsis thaliana. Mol Gen Genet 262, 633-642.

Kong, X., Lu, S., Tian, H., and Ding, Z. (2015). WOX5 is shining in root stem cell niche. Trends Plant Sci 20, 601-603.

Kunarso, G., Chia, N.Y., Jeyakani, J., Hwang, C., Lu, X., Chan, Y.S., Ng, H.H., and Bourque, G. (2010). Transposable elements have rewired the core regulatory network of human embryonic stem cells. Nat Genet 42 , 631-634.

Lafarge, S., and Montane, M.H. (2003). Characterization of Arabidopsis thaliana ortholog of the human breast cancer susceptibility gene 1: AtBRCA1, strongly induced by gamma rays. Nucleic Acids Res 31, 1148-1155

Li, H., Ilin, S., Wang, W., Duncan, E., Wysocka, J., Allis, C., and Patel, D. (2006). Molecular basis for site-specific read-out of histone H3K4me3 by the BPTF PHD finger of NURF. Nature 442, 91-95.

Lu, F., Cui, X., Zhang, S., Jenuwein, T., and Cao, X. (2011). Arabidopsis REF6 is a histone H3 lysine 27 demethylase. Nat. Genet 43, 715-719.

Reidt, W., Wurz, R., Wanieck, K., Chu, H.H., and Puchta, H. (2006). A homologue of the breast cancer-associated gene BARD1 is involved in DNA repair in plants. EMBO J 25, 4326-4337.

Sage, J.(2012). The retinoblastoma tumor suppressor and stem cell biology. Genes Dev 26, 1409-1420.

Scheres, B. (2007). Stem-cell niches: nursery rhymes across kingdoms. Nat Rev Mol Cell Biol 8, 345-354.

Shi, X., Hong, T., Walter, K., Ewalt, M., Michishita, E., Hung, T., Carney, D., Pena, P., Lan, F., Kaadige, M., Lacoste, N., Cayrou, C., Davrazou, F., Saha, A., Cairns, B., Ayer, D., Kutateladze, T., Shi, Y., Cote, J., Chua, K., and Gozani, O. (2006). ING2 PHD domain links histone H3 lysine 4 methylation to active gene repression. Nature 442, 96-99.

Soltis, P.S., Soltis, D.E., Savolainen, V., Crane, P.R., and Barraclough, T.G. (2002). Rate heterogeneity among lineages of tracheophytes: integration of molecular and fossil data and evidence for molecular living fossils. Proc Natl Acad Sci USA 99, 4430-4435.

Song, X., Yu, D., Xu, T., Ren, S., Guo, P., and Liu, C. (2012) Contributions of individual amino acid residues to the endogenous CLV3 function in shoot apical meristem maintenance in Arabidopsis. Mol Plant 5, 515-523

Sung, S., and Amasino, R. (2004). Vernalization in Arabidopsis thaliana is mediated by the PHD finger protein VIN3. Nature 427, 159-164.

Su, Y., Liu, Y., and Zhang, X. (2011). Auxin-cytokinin interaction regulates meristem development. Mol Plant 4, 616-625.

Su, Y., Zhao, X., Liu, Y., Zhang, C., O’Neill, S.D, and Zhang, X. (2009) Auxin-induced WUS expression is essential for embryonic stem cell renewal during somatic embryogenesis in Arabidopsis. Plant J 59, 448-460.

Swoboda, P., Gal, S., Hohn, B., and Puchta, H. (1994). Intrachromosomal homologous recombination in whole plants. EMBO J 13, 484-489.

Trapp, O., Seeliger, K., and Puchta, H. (2011). Homologs of breast cancer genes in plants. Front Plant Sci doi: 10.3389/fpls.2011.00019. Wang, K., Huang, G., and Zhu, Y.X. (2016). Transposable elements play an important role during cotton genome evolution and fiber cell development. Sci China Life Sci 59, 112-121.

Waterworth, W.M., Altun, C., Armstrong, S.J., Roberts, N., Dean, P.J., Young, K., Weil, C.F., Bray, C.M., and West, C.E. (2007). NBS1 is involved in DNA repair and plays a synergistic role with ATM in mediating meiotic homologous recombination in plants. Plant J 52, 41-52.

Wei, L. and Cao, X. (2016). The effect of transposable elements on phenotypic variation: insights from plants to humans. Sci China Life Sci 59, 24-37.

Weigel, D., and Jürgens, G. (2002). Stem cells that make stems. Nature $415,751-754$.

Westermark, U.K., Reyngold, M., Olshen, A.B., Baer, R., Jasin, M., and Moynahan, M.E. (2003). BARD1 participates with BRCA1 in homology directed repair of chromosome breaks. Mol Cell Biol 23, 7926-7936.

Williams, R.S., Lee, M.S., Hau, D.D., and Glover, J.N. (2004). Structural basis of phosphopeptide recognition by the BRCT domain of BRCA1. Nat. Struct Mol Biol 11, 519-525.

Wu-Baer, F., Lagrazon, K., Yuan, W., and Baer R. (2003). The BRCA1/BARD1heterodimer assembles polyubiquitin chains through an unconventional linkage involving lysine residue $\mathrm{K} 6$ of ubiquitin. J Biol Chem 278, 34743-34746.

Wysocka, J., Swigut, T., Xiao, H., Milne, T., Kwon, S.Y., Landry, J., Kauer, M., Tackett, A.,Chait, B., Badenhorst, P., Wu, C., and Allis, C. (2006). A PHD finger of NURF couples histone H3 lysine 4 trimethylation with chromatin remodelling. Nature 442, 86-90.

Xu, L. (2015). Meristem control of leaf patterning. Sci China Life Sci 58, $315-316$.

Xu, T.T., Song, X.F., Ren, S.C., and Liu, C.M. (2013) The sequence flanking the $\mathrm{N}$-terminus of the CLV3 peptide is critical for its cleavage and activity in stem cell regulation in Arabidopsis. BMC Plant Biol 13, 225.

Yang, S., Li, C., Zhao, L., Gao, S., Lu, J., Zhao, M., Chen, C.Y., Liu, X., Luo, M., Cui, Y. et al. (2015). The Arabidopsis SWI2/SNF2 chromatin remodeling ATPase BRAHMA targets directly to PINs and is required for root stem cell niche maintenance. Plant Cell 27, 1670-1680.

Yoon, H.S., Hackett, J.D., Ciniglia, C., Pinto, G., and Bhattacharya, D. (2004). A molecular timeline for the origin of photosynthetic eukaryotes. Mol Biol Evol 21, 809-818.

Zhang, Y., Jiao, Y., Liu, Z., and Zhu, Y.X. (2015). ROW1 maintains quiescent centre identity by confining WOX5 expression to specific cells. Nat Commun doi: 10.1038/ncomms7003.

Zhou, Y., Liu, X., Engstrom, EM., Nimchuk Z.L., Pruneda-Paz, J.L., Tarr, P.T., Yan, A., Kay, S.A., and Meyerowitz, E.M. (2015). Control of plant stem cell function by conserved interacting transcriptional regulators. Nature 517, 377-380.

Zimmer, A., Lang, D., Richardt, S., Frank, W., Reski, R., and Rensing, S.A. (2007). Dating the early evolution of plants: detection and molecular clock analyses of orthologs. Mol Genet Genomics 278, 393-402.

Open Access This article is distributed under the terms of the Creative Commons Attribution License which permits any use, distribution, and reproduction in any medium, provided the original author(s) and source are credited. 